\title{
Estimation of the thermal diffusivities tensor out of the main axes of anisotropy with a pulsed laser spot method
}

\author{
by L. Gavérina*, J.C Batsale*, D. Mourand** and C. Pradere*
}

* I2M-TREFLE, UMR CNRS 5295, Esplanade des arts et métiers 33405 Talence Cedex, France, ludovic.gaverina@u-bordeaux.fr; jean-christophe.batsale@u-bordeaux.fr; c.pradere@i2m.u-bordeaux1.fr; **Epsilon Fahrenheit, ENSAM, Esplanade des Arts et Métiers, F-33405 Talence Cedex, France dmourand@epsilon-alcen.com

\begin{abstract}
Several methods have been proposed in order to measure the in-plane thermal diffusivities along the main axes of orthotropic materials. To our knowledge, few methods exist in order to simultaneously estimate the thermal diffusivities tensor out of the main axes of anisotropy, and the position of the main axes. In this paper we proposed a thermal ellipsometry method based on a pulsed laser spot method and an inverse processing method to estimate the thermal diffusivities tensor from the recording of the transient thermal field at the front face.
\end{abstract}

\section{Introduction}

In the aerospace industry, the implementation of composite material is constantly increasing. During the elaboration process of these materials, it is important to survey the arrangement or orientation of the fibers to control the thermal properties and their strength. Many techniques exist to verify the quality of fiber organisation by using destructive methods. Nevertheless, the used of Non Destructive Technique (NDT) is a powerful tool for online monitoring and a quantitative and fast characterization.

One century ago, De Sénarmont [1] proposed a thermal approach to estimate the principal orientation in crystal plates. The results of this work show that isotherms are elliptical and that aspect ratio was equal to the square root of the principal conductivities in the surface plane. Later, Krapez et al. [2], developed a "Thermal Ellipsometry" method by using lock-in thermography technique applied to polymer material by estimating the correlation between their draw ratio and the induced thermal anisotropy when the main axes are assumed well-known [3]. Whatever, one major problem of such anisotropic material is the knowledge of the main axes that is generally not easy to retrieve. For these reasons, in this paper, a novel method is proposed in order to improve the thermal characterization of anisotropic material out of the main axes of anisotropy.

In this work, the analysis of the front face temperature response field after front face pulsed laser spot is proposed. It is shown that many parameters could be estimated as: (i), the anisotropic ratio, (ii), the thermal diffusivities tensor and (iii), the initial position of the spot. The main interest of the pulsed spot method [4] is the quick implementation of a real time characterization of a complex thermal problem in anisotropic materials.

\section{Presentation of the method for a simple case}

\subsection{Analytical modelization of the problem}

The analytical expression of the front face temperature response field of a pulsed source point located in ( $x_{0}, y_{0}$ and $z$ $=0$ ), of a homogeneous and anisotropic (with a main axis following the $z$ direction) medium [5][6][7] could be written as follows:

$$
T(x, y, z=0, t)=\frac{Q}{4 \pi \rho C_{p} t} *\left|a^{i, j}\right|^{\frac{1}{2}} \exp \left(-\left(\frac{a^{x x}\left(x-x_{0}\right)^{2}}{4 t}+\frac{a^{x y}\left(x-x_{0}\right)\left(y-y_{0}\right)}{2 t}+\frac{a^{y y}\left(y-y_{0}\right)^{2}}{4 t}\right)\right) * f_{z}(t)
$$

Where:

- $\quad a^{x x}, a^{x y}, a^{y y}$ are the inverse thermal diffusivities tensor defined by $a^{i, j}=\frac{1}{\left|a_{i, j}\right|}\left(\begin{array}{cc}a_{y y} & -a_{x y} \\ -a_{x y} & a_{x x}\end{array}\right),\left(\mathrm{m}^{2} . \mathrm{s}^{-1}\right)$

- $\left|a^{i, j}\right|$ is the determinant of the inverse thermal diffusivities tensor, $\left(\mathrm{m}^{2} \cdot \mathrm{s}^{-1}\right)$

- $\quad x_{0}, y_{0}$ : in-plane spatial position of the laser spot versus $x, y$ direction $(\mathrm{m})$

- $Q$ : energy density of the pulse point source $(\mathrm{J})$

- $\quad \rho$ : density $\left(\mathrm{k}_{\mathrm{g}} \cdot \mathrm{m}^{-3}\right)$

- $\quad C_{p}$ : specific heat capacity $\left(\mathrm{J} \cdot \mathrm{kg}^{-1} \cdot \mathrm{K}^{-1}\right)$

- $f_{z}(t)$ is the evolution of the spatially averaged field at the front face $(z=0)$ of the sample 


\subsection{1/qirt.2016.037}

If the sample is semi-infitnite versus the $z$-direction, the $f z$ transient behavior is inversely proportional to the square root of the time and the Eq. (1) can be written as function of the thermal diffusivities tensor, as follow:

$$
T(x, y, z=0, t)=\frac{Q}{4 \pi \rho C_{p} t} * \frac{\left|a^{i, j}\right|^{\frac{1}{2}}}{\sqrt{\pi a_{z} t}} \exp \left(-\frac{1}{\left|a_{i, j}\right|}\left(\frac{a_{y y}\left(x-x_{0}\right)^{2}}{4 t}-\frac{a_{x y}\left(x-x_{0}\right)\left(y-y_{0}\right)}{2 t}+\frac{a_{x x}\left(y-y_{0}\right)^{2}}{4 t}\right)\right)
$$

With $a_{i, j}$ is thermal diffusivities tensor defined by $a_{i, j}=\left(\begin{array}{ll}a_{x x} & a_{x y} \\ a_{x y} & a_{y y}\end{array}\right)$ and $\left|a_{i, j}\right|$ is his determinant.

The consideration of the logarithm of the Eq. (2) yields to the Eq. (3) of an ellipse centered at $\left(\mathrm{x}_{0}, \mathrm{y}_{0}\right)$. By using a linear least squares [4] inverse processing, the coefficients of this quadratic form are estimated.

At a fixed time $t$, the isothermal lines given by the Eq. (3) are the exact quadratic form of an ellipse which is very suitable in order to estimate the thermal diffusivities tensor, the center of the ellipse and the off-axis orientation.

$$
\ln (T(x, y, z=0, t))=\ln \left(\frac{Q}{4 \pi \rho C_{p} t} * \frac{\left|a^{i, j}\right|^{\frac{1}{2}}}{\sqrt{\pi a_{z} t}}\right)+\frac{1}{\left|a_{i, j}\right|}-\frac{a_{y y}}{4 t}\left(x-x_{0}\right)^{2}+\frac{a_{x y}}{2 t}\left(x-x_{0}\right)\left(y-y_{0}\right)-\frac{a_{x x}}{4 t}\left(y-y_{0}\right)^{2}
$$

The expression can be assimilated to the following elliptical relation:

$$
\ln (T(x, y, z=0, t))=k_{0}(t)+k_{1}(t) x+k_{2}(t) y+k_{3}(t) x^{2}+k_{4}(t) x y+k_{5}(t) y^{2}
$$

Where:

$$
\Leftrightarrow\left\{\begin{array}{l}
k_{0}(t)=\ln \left(\frac{Q}{4 \pi \rho C_{p} t} * \frac{\left|a^{i, j}\right| \frac{1}{2}}{\sqrt{\pi a_{z} t}}\right)+\frac{1}{\left|a_{i, j}\right|}-\frac{a_{y y}(t)}{4 t} x_{0}^{2}+\frac{a_{x y}(t)}{2 t} x_{0} y_{0}-\frac{a_{x x}(t)}{4 t} y_{0} \\
k_{1}(t)=-\frac{a_{x y}(t) y_{0}}{2 t}+\frac{a_{y y}(t)}{2 t} x_{0} \\
k_{2}(t)=-\frac{a_{x y}(t)}{2 t} x_{0}+\frac{a_{x x}(t)}{2 t} y_{0} \\
k_{3}(t)=-\frac{a_{y y}(t)}{4 t} \\
k_{4}(t)=\frac{a_{x y}(t)}{2 t} \\
k_{5}(t)=-\frac{a_{x x}(t)}{4 t}
\end{array}\right.
$$

Several comments arise from the previous expressions:

- $\quad k_{3}(t), k_{4}(t), k_{5}(t)$ depend respectively on the thermal diffusivities $a_{y y}, a_{x y}, a_{x x}$. Thus, the in-plane homogenous transfer at different time steps could be verified, independently of the transverse heat transfer and the laser spot position.

- When the thermal diffusivities are estimated using $k_{3}(t), k_{4}(t), k_{5}(t)$, the position of the initial spot $x_{0}$ and $y_{0}$ could be calculated by using the $k_{2}(t)$ and $k_{1}(t)$ parameters. 


\subsection{1/qirt.2016.037}

\subsection{Estimation of the parameters}

Because in experimentation the set of measured data are noisy, a noise is considered for the following estimation method. As a consequence, an uniform noise is added to the temperature as follows:

$$
\hat{T}\left(x, y, z=0, t_{i}\right)=T\left(x, y, z=0, t_{i}\right)+e_{T\left(x, y, z=0, t_{i}\right)}
$$

with $e_{T(x, y, t)}$ random fluctuation added to a signal $T\left(x, y, z=0, t_{i}\right)$. It is considered with a zero mean and uniform standard deviation (covariance matrix is diagonal).

$$
\ln \left(\hat{T}\left(x, y, z=0, t_{i}\right)=\ln \left(T\left(x, y, z=0, t_{i}\right)\right)+\frac{e_{T\left(x, y, z=0, t_{i}\right)}}{T\left(x, y, z=0, t_{i}\right)}\right.
$$

After a logarithm decomposition of the Eq. (7), the noise which is not uniform will highly increases when the signal $T\left(x, y, z=0, t_{i}\right)$ is low in expression (7). As a consequence it becomes very important to improve the thresholding of the less noisy signal $T\left(x, y, z=0, t_{i}\right)$. Such tresholding will be implemented when the noise is about $10 \%$ of the maximum signal on the instantaneous temperature image.

From the measurements point of view, a quadratic fitting can be implemented at each time $t$. The parameters $k_{n}(t)$ can be estimated by using a linear least-square relationship with a Gauss-Markov inverse method. The Eq. (4) can be expressed as a linear combination of the logarithm of the measured temperature $\ln \left(\widehat{T}\left(x, y, z=0, t_{i}\right)\right.$ and the $k_{n}(t)$ parameters, $\ln \left(\hat{T}\left(x, y, z=0, t_{i}\right)=X . \hat{\beta}\right.$, where $\mathrm{X}$ is the sensitivity matrix.

One estimator yields then:

$$
\left[\begin{array}{l}
\hat{k}_{0}(t) \\
\hat{k}_{1}(t) \\
\hat{k}_{2}(t) \\
\hat{k}_{3}(t) \\
\hat{k}_{4}(t) \\
\hat{k}_{5}(t)
\end{array}\right]=\left(X^{t} X\right)^{-1} X^{t}[\ln (\hat{T})]
$$

where:

$$
X=\left[\begin{array}{cccccc}
1 & x & y & x y & x^{2} & y^{2} \\
\vdots & \vdots & \vdots & \vdots & \vdots & \vdots \\
1 & x_{i} & y_{i} & x_{i} y_{i} & x_{i}{ }^{2} & y_{i}{ }^{2}
\end{array}\right]
$$

\section{Validation of the method on experimental data}

\subsection{Experimental set-up}

The laser used in this set-up is an IR diode of $976 \mathrm{~nm}$ wavelength and a power of $330 \mathrm{~mW}$. An optical system (Thorlabs) is used to collimate the laser beam. A Dual-Axis Scanning Galvo System (Thorlabs GVS112/M) was used to control the spatial displacement of a laser hot spot over the in-plane surface $(z=0)$ of the sample. The principle of the laser beam deviation towards the sample is shown below (figure 1). To focus the laser beam on the surface, a f-theta scan lens was used. The focus length of this lens is $192 \mathrm{~mm}$, and the resulting diameter of the focused spot is $26 \mu \mathrm{m}$. In order that the focalized spot will be perfectly perpendicular to the surface of the sample, a dichroic mirror mounted at $45^{\circ}$ is used. As a consequence, the incident beam is normal to the sample surface and the IR camera as well. This laser diode is mounted horizontally and the beam is reflected with the dichroic mirror (MD, treated to reflect the visible light from $700 \mathrm{~nm}$ to $1000 \mathrm{~nm}$ and to transmit the infrared radiation (95\% of transmission between 2 to $5 \mu \mathrm{m})$ ). To measure the temperature fields, an IR camera MCT (FLIR SC7000, 320*256 pixels, pitch $30 \mu \mathrm{m}$, spectral band from 7 to $14 \mu \mathrm{m}$ ) was used with an infrared objective lens (focal $25 \mathrm{~mm}$ ). Finally, the resulting spatial resolution is around $200 \mu \mathrm{m}$ per pixel. 


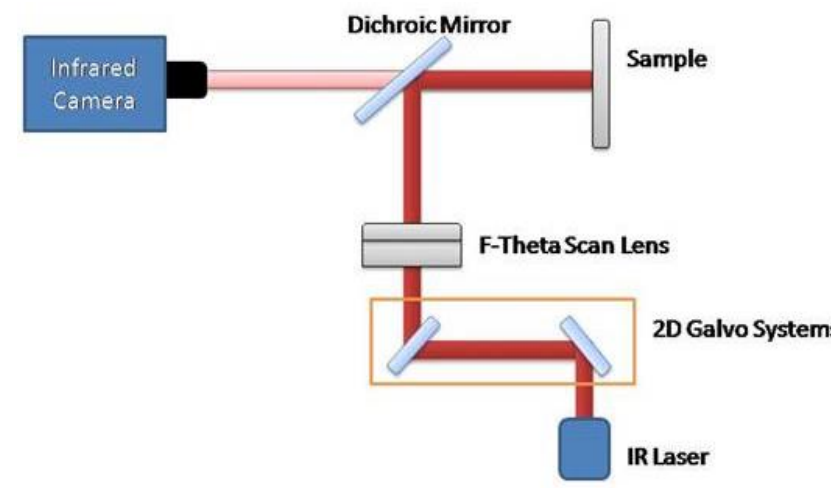

a)

b)

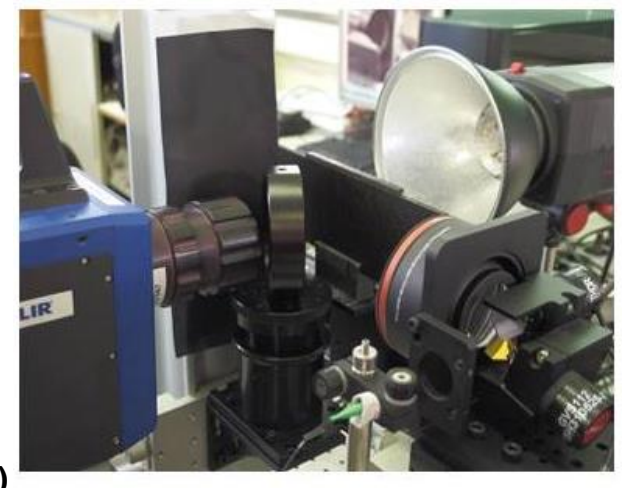

Fig. 1. Experimental set-up: a, scheme of the system and b, photography

\subsection{Estimation of the thermal diffusivities tensor out of the main axes of anisotropy}

An experimental validation was performed with the previous set-up and with the optical alignment we used, the spatial resolution was approximately $118 \mu \mathrm{m}$ per pixel. The frame rate acquisition was fixed at $100 \mathrm{~Hz}$. The sample to characterize was a carbon/epoxy composite material with a thickness of $5 \mathrm{~mm}$ and in-plane dimension of $L=236 \mathrm{~mm}$ and I = $75 \mathrm{~mm}$. The transverse thermal diffusivity (in direction) was already estimated in previous study [8], and its value is around $4.63^{*} 10^{-7} \mathrm{~m}^{2} \cdot \mathrm{s}^{-1}$.

a)

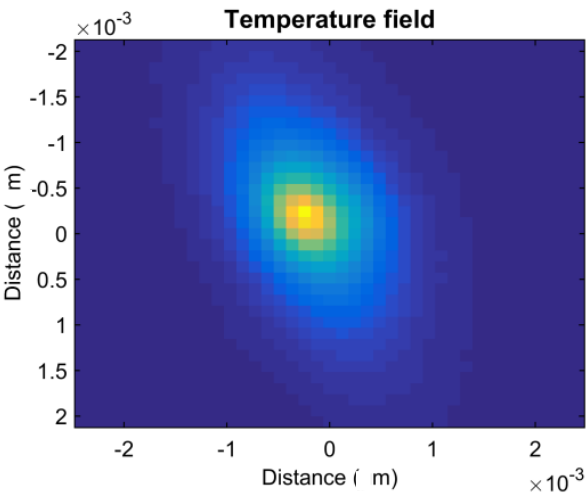

c)

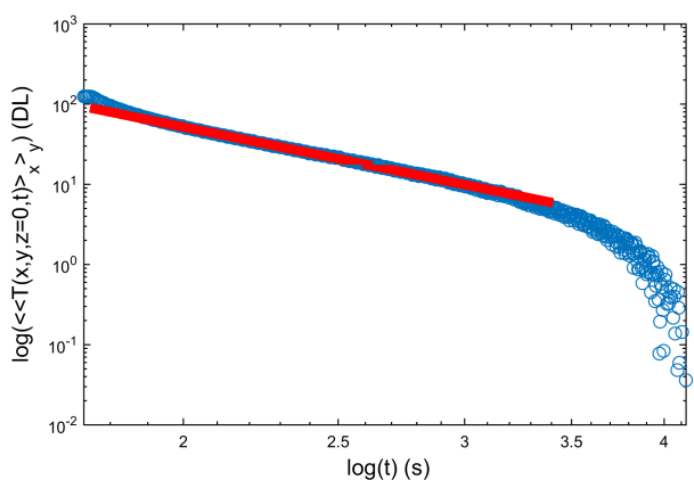

b)

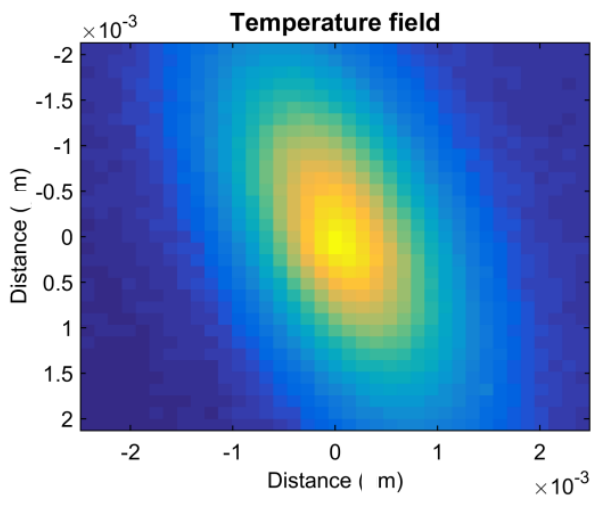

Fig. 2. a), Measured temperature just after the laser pulse. b), Temperature profile along the major and minor axes of the ellipse just after the laser pulse. c), The logarithm of spatial average value of the temperature field (blue circle) is plotted versus logarithm of time. The $-1 / 2$ slope (red line) is well observed until $t=3.5 \mathrm{~s}$, than signal-to-noise ratio is low 


\subsection{1/qirt.2016.037}

Based on this IR measurement, the estimations of the parameters $\mathrm{k}_{\mathrm{i}}(\mathrm{t})$ of the thermal diffusivities tensor are plotted in figure 3. As illustrated in figure 3, the estimation of the thermal diffusivities tensor gives:

$a_{x x}=1 \cdot 61 \cdot 10^{-6} \mathrm{~m}^{2} \cdot \mathrm{s}^{-1}, a_{y y}=3 \cdot 44 \cdot 10^{-7} \mathrm{~m}^{2} \cdot \mathrm{s}^{-1}$ and $\mathrm{a}_{\mathrm{xy}}=3 \cdot 61 \cdot 10^{-7} \mathrm{~m}^{2} \cdot \mathrm{s}^{-1}$. Such results are improving the performances of classical thermal ellipsometry method because it is possible to estimate the thermal diffusivities tensor out of the main axes of anisotropy with a good accuracy.

At short time, the estimation is biased because the temporal shape of the spot is not a perfect Dirac but a square of $500 \mathrm{~ms}$. As a consequence, the measured temperature is convoluted with and could be expresses as follow: $\tilde{T}(x, y, z=0, t)=T(x, y, z=0, t) * \Pi(\tau)$. After a long time, the noise from the measurement increases during the relaxation time. As a consequence, the error in both the estimation of the thermal diffusivity along both directions increased. The best estimation part corresponds to the thermal relaxation, where linear behavior is observed with a high signal-to-noise ratio.

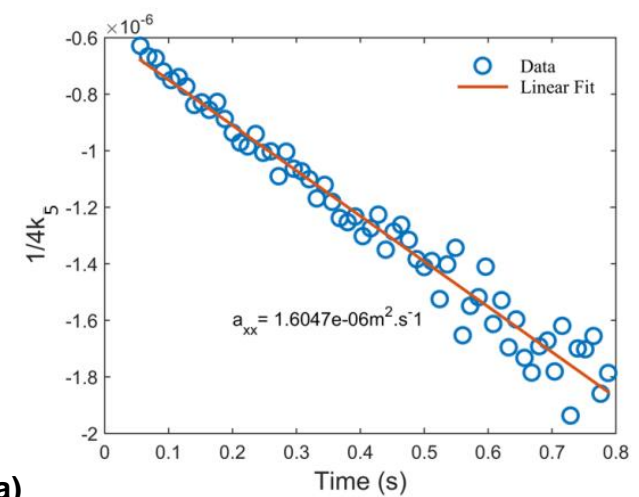

a)

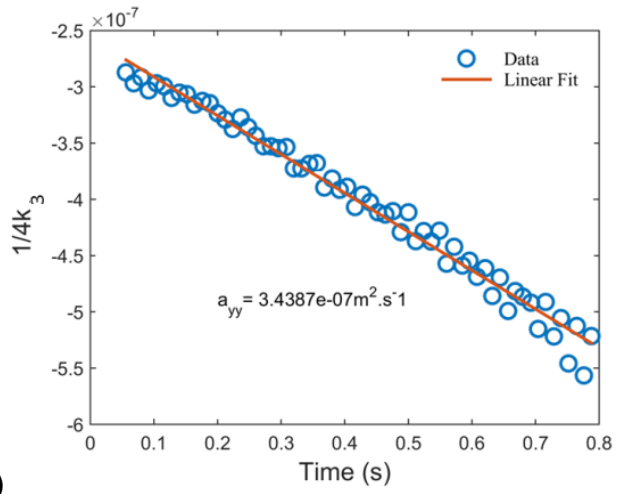

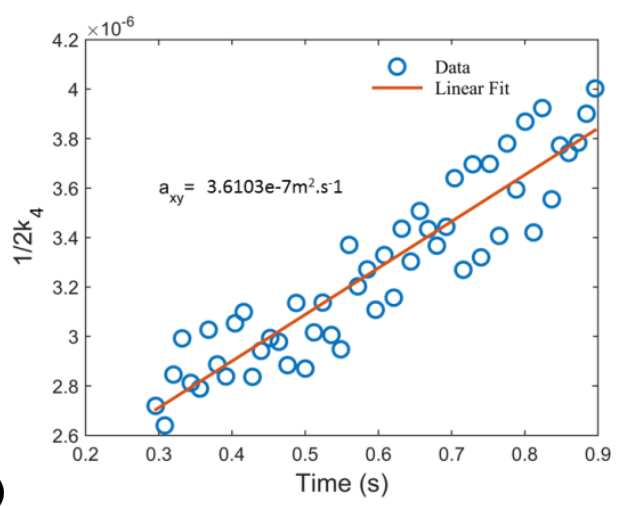

b)

Fig. 3. Estimation of the thermal diffusivities tensor: a) along the $x$ direction $k_{5}$ parameter, b) along the $x y$ direction $k_{4}$ parameter and c) along the $y$ direction $k_{3}$ parameter.

\subsection{Recovering of the main parameters}

For any conics the algebraic expression is dependent on the location and orientation of the coordinate system and the conics have the form of a second-degree quadratic. This kind of quadratic expression is difficult to retrieve because of the mixed term in $x y$. It is possible to simplify this expression by eliminating the mixed term xy. For that, a simple shift of the canonical basis to eigenvector basis could be performed. As a consequence the expression Eq. (4) could be rewritten as follow:

$$
\ln \left(T\left(x^{\prime}, y^{\prime}, t\right)\right)=C(t)+k_{3}(t) x^{\prime 2}+k_{4}(t) x^{\prime} y^{\prime}+k_{5}(t) y^{\prime 2}
$$

Where $x^{\prime}$ and $y^{\prime}$ are respectively equal to $x-x_{0}$ and $y-y_{0}$. 


\subsection{1/qirt.2016.037}

The general second degree equation can be reduced to one of the following canonical forms:

$$
q^{\prime}(X, Y)=\alpha_{1} X^{2}+\alpha_{2} Y^{2}
$$

From Eq. (9) the ellipse could be recalculated in the main axes of anisotropy by using a base shift (figure 5). To realize such shift, several steps are necessary:

(1) Inverse the equation of ellipse: $q\left(x^{\prime}, y^{\prime}\right)={ }^{t} X A X$, where $X=\left(\begin{array}{l}x^{\prime} \\ y^{\prime}\end{array}\right)$ and A the associated matrix

$$
\left(\begin{array}{cc}
k_{3} & \frac{k_{4}}{2} \\
\frac{k_{4}}{2} & k_{5}
\end{array}\right)
$$

(2) Study of the associated matrix $\left(\begin{array}{cc}k_{3} & \frac{k_{4}}{2} \\ \frac{k_{4}}{2} & k_{5}\end{array}\right)$ to estimate eigenvalues $\left(\frac{1}{a_{x}}, \frac{1}{a_{y}}\right)$ and eigenvectors $\left(\overrightarrow{e_{1}}, \overrightarrow{e_{2}}\right)$.

(3) Recovering of the main parameters: shift of anisotropic axes: $A=P D P^{-1}$ where $D$ is diagonal matrix and $P$ change of basis matrix.

New quadratic form of the main axes of anisotropy: $q^{\prime}(X, Y)=\left(\begin{array}{ll}X & Y\end{array}\right)\left(\begin{array}{cc}\frac{1}{a_{x}} & 0 \\ 0 & \frac{1}{a_{y}}\end{array}\right)\left(\begin{array}{l}X \\ Y\end{array}\right)=\frac{X^{2}}{a_{x}}+\frac{Y^{2}}{a_{y}}$

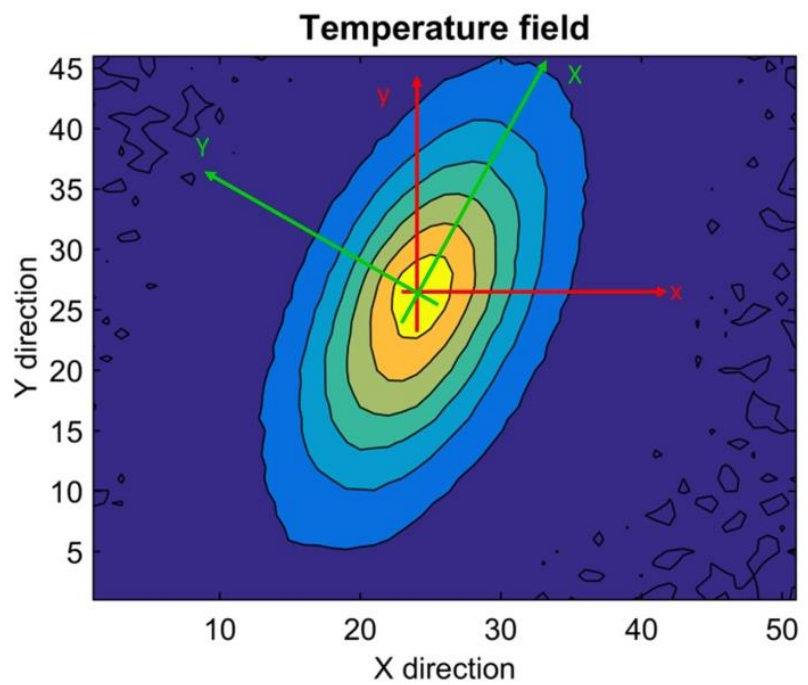

Fig. 5. The experimental temperature field during the thermal relaxation (isothermal lines) of a composite material and shift of the temperature field (green axes) out of the main anisotropic axes (red axes).

It is possible to obtain the novel expression of the temperature response field of a pulsed source point, imposed on the front face $(z=0)$ for the front surface of a homogeneous and anisotropic semi-infinite media in the in the main axes (eigenvector basis) of anisotropy after applying the different processing (see part 2) as follows:

$$
T(x, y, z=0, t)=\frac{Q}{4 \rho C_{p}} \frac{\exp \left(-\frac{\left(X-x_{0}\right)^{2}}{4 a_{x} t}\right)}{\sqrt{\pi a_{x} t}} \frac{\exp \left(-\frac{\left(Y-y_{0}\right)^{2}}{4 a_{y} t}\right)}{\sqrt{\pi a_{y} t}} f_{z}(t)
$$




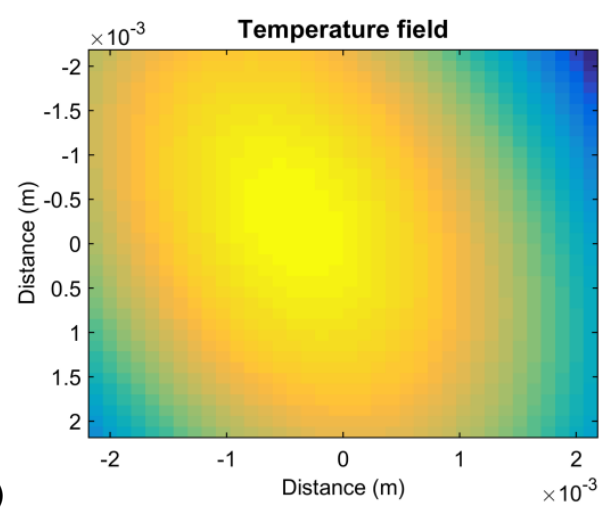

Fig. 6. The shift of the experimental temperature field from a canonical basis to eigenvector ba: is after eliminating of the mixed term xy: a) The ellipse out of the main axes of anisotropy and b) The ellipse in the main axes of anisotropy.

From the change of basis matrix, it is possible to retrieve the angle of rotation of the main anisotropic directions. In this case, the angle of rotation is $-19^{\circ}$.

\section{Conclusion}

The first results presented in this paper are devoted to improve the classical thermal ellipsometry technique by the proposition of a new method based on the thermal diffusivities tensor estimation. This new method is based on a front face pulsed thermography method and the estimation of the tensor of thermal diffusivities by fitting the logarithm of the measured data with the equation of an ellipse. First results presented on an homogenous and anistropic sample of Carbone/Epoxy demonstrate that there is no obstacle for thermal characterization of anisotropic material out of the main axis of anisotropy. Moreover, by using the polar properties of such an elliptic equation, it becomes possible to retrieve the angle of rotation of the main anisotropic directions.

In the future, the recovering of the main axes can be used in order to detect if the sample have a rough surface or if the shape of spot laser is elliptical. The perspective of this study is to test the method in several situations, interfaces, mapping of thermophysical properties, and research of a small anomaly over a large area.

\section{REFERENCES}

[1] H. H. de Sénarmont, "Mémoire sur la conductibilité des substances cristalisées pour la chaleur," C. r. Hebd. Séance Acadamie Sci. Paris, vol. 25, pp. 459-461, 1848.

[2] J.-C. Krapez and G. Gardette, "Characterisation of anisotropic materials by steady-state and modulated thermal ellipsometry," High Temp. High Press., vol. 30, no. 5, pp. 567-574, 1998.

[3] H.-C. Fernandes and X. Maldague, "Fiber orientation assessment in complex shaped parts reinforced with carbon fiber using infrared thermography," Quant. InfraRed Thermogr. J., vol. 12, no. 1, pp. 64-79, 2015.

[4] L. Gavérina, J.-C. Batsale, D. Mourand, and C. Pradère, "Several considerations about a pulsed flying spot method implemented with IR thermography," Quant. InfraRed Thermogr., Jul. 2015.

[5] H. S. Carslaw and J. C. Jaeger, "Conduction of heat in solids," Oxf. Clarendon Press 1959 2nd Ed, 1959.

[6] B. T. Chao, "A note on conduction of heat in anisotropic media," Appl. Sci. Res. Sect. A, vol. 12, no. 2, pp. 134-138, 1963.

[7] Y. P. Chang, C. S. Kang, and D. J. Chen, "The use of fundamental Green's functions for the solution of problems of heat conduction in anisotropic media," Int. J. Heat Mass Transf., vol. 16, no. 10, pp. 1905-1918, 1973.

[8] M. Thomas, "Propriétés thermiques de matériaux composites: caractérisation expérimentale et approche microstructurale," Nantes, 2008. 